\title{
LOW TEMPERATURE ABSORPTION SPECTROSCOPY OF BARLEY MUTANTS. GUASSIAN DECONVOLUTION AND FOURTH DERIVATIVE ANALYSIS
}

by

\author{
DAVID J. SIMPSON \\ Department of Physiology, Carlsberg Laboratory, \\ Gamle Carlsberg Vej 10, DK-2500 Copenhagen Valby
}

Keywords: Chlorophyll-proteins, photosystem I/II, thylakoids

\begin{abstract}
The absorption spectra of wild type barley and a number of nuclear gene mutants have been recorded at $77 \mathrm{~K}$, and analysed by taking the fourth derivative and Gaussian deconvolution in the region $600-725 \mathrm{~nm}$. Seven Gaussian curves could be fitted, corresponding to the $\mathrm{Q}_{\mathrm{y}}$ transitions of at least 7 different chlorophyll species, absorbing at 640.5 and $649.9 \mathrm{~nm}$ (chlorophyll $b$ ), 660.7, 668.9, 676.6, 682.9 and $688.9 \mathrm{~nm}$ (chlorophyll $a$ ). The first 2 components were missing from the chlorophyll $b$-less mutant $c l o-\Omega^{2800}$, revealing a minor chlorophyll $a$ species at $653 \mathrm{~nm}$. The same 7 wild type components could also be fitted to the low temperature absorption spectra of the mutants vir- $m^{29}$ and $v i r-k^{23}$. The loss of photosystem II from the mutant $v i r-113$ was associated with the specific loss of the $683 \mathrm{~nm}$ component, corresponding to about 30-40 molecules of chlorophyll $a$ per 500 molecules of chlorophyll. Similarly, the absence of photosystem I from the mutant vir-z $z b^{63}$ was correlated with the loss of 30 molecules of chlorophyll $a$ absorbing at $689 \mathrm{~nm}$. These specific differences were confirmed by the absorption difference spectra and by fourth derivative spectroscopy. The validity of the solutions provided by Gaussian deconvolution were evaluated by comparing their fourth derivative with that of the original spectra.
\end{abstract}

\section{INTRODUCTION}

About half of the protein in chloroplasts, and almost all of the lipids, are found in the thylakoid membranes. Of the lipids, which comprise $37 \%$ of the dry weight of thylakoids (11), chlorophyll $a$ and $b$ account for $21.5 \%$, equivalent to $8 \%$ of the dry weight. Although this represents a protein:chlorophyll ratio of 6.3:1, isolated chlorophyll-proteins have even higher concentrations of chlorophyll, with protein: chlorophyll ratios of 3:1 for P700 $\mathrm{Chl}_{a}-\mathrm{P} 1$ (19) or 2.2:1 for LHCII (12). Assuming a protein density of $1.13 \mathrm{~g} \cdot \mathrm{cm}^{-3}$, this is equivalent to a chlorophyll $a$ concentration of between 0.33 $0.42 \mathrm{M}$. All of the chlorophyll is non-covalently associated with the polypeptide chain, since it can be removed by solvent extraction, but very little is known about the nature of the ligands involved in binding chlorophyll, or the structure of the different chlorophyll-proteins.

The absorption spectrum of chlorophyll $a$ is due to the capture of photons with the correct

Abbreviations: $\mathrm{CD}=$ circular dichroism; $\mathrm{Chl}=$ chlorophyll; clo = chlorina CPI = chlorophyll-protein I; LHCI/II $=$ light-harvesting chlorophyll $a / b$-protein of $\mathrm{PSI} / \mathrm{II}$; PSI $/ \mathrm{II}=$ photosystem $\mathrm{I} / \mathrm{II}$; Tricine $=\mathrm{N}$-(tris(hydroxymethyl)methyl)-glycine; vir = viridis. 


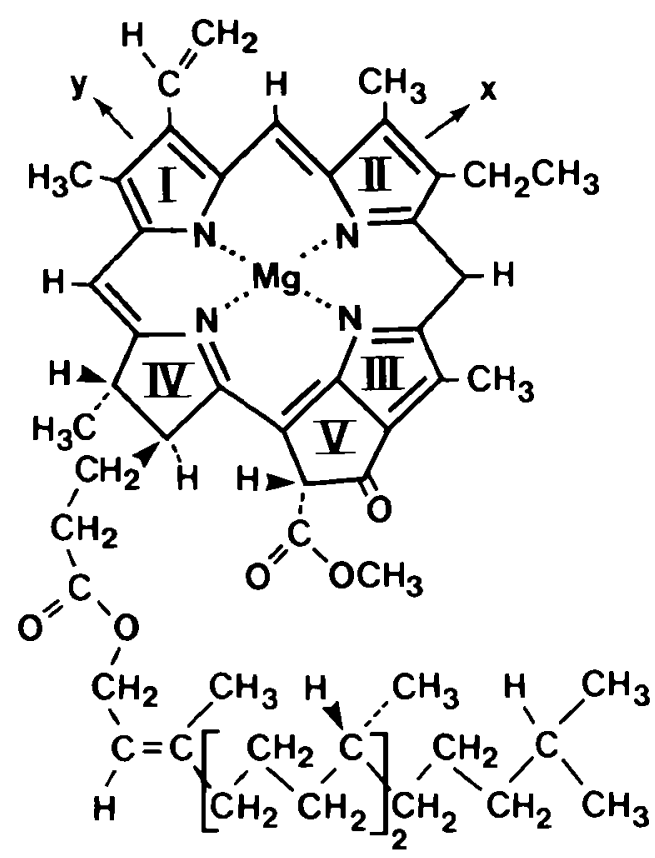

CHLOROPHYLLa

Figure 1. Molecular structure of chlorophyll $a$ with the convention for the $\mathrm{x}$ and $\mathrm{y}$ directions within the plane of the porphyrin ring indicated by arrows. The $\pi$ electrons of the conjugated porphyrin rings are responsible for the absorption of light in the visible spectrum.

energy to raise the chlorophyll molecule from the ground state into one of several "allowed" excited electronic states (25). These arise from $\pi$ $-\pi$ electronic interactions within the conjugated porphyrin ring system (Fig. 1) $(11,25)$. The energy levels are rather broad due to excitation of molecular vibrations as well as the electrons, and the main absorption bands are designated $B_{x}, B_{y}$ (Soret band) and $Q_{x}$ and $Q_{y}$. For chlorophyll $a$ in solution, the lowest energy transition occurs at $660 \mathrm{~nm}$ in the $Q_{y}$ region, while the $Q_{x}$ band lies at $575 \mathrm{~nm}(25)$.

The chlorophyll $\mathrm{Q}_{\mathrm{y}}$ absorption band is much more complex in vivo than in vitro, and up to 8-10 components have been resolved by fourth derivative spectroscopy $(3,4,7,15,16,24)$ and Gaussian deconvolution $(2-5,8-10,14,18,20$, $22,24)$ of thylakoids. Since chlorophyll is chemically unaltered in the membrane, these diffe- rent forms must arise from the different environments in which the chlorophyll molecules occur in vivo. It is unlikely that the different $Q_{y}$ absorption bands come from oligomeric states of chlorophyll $a$ since these require the rigorous exclusion of water for their formation (11). Alternatively, the $\mathrm{Mg}$ atom may be coordinated to different polypeptide side chain ligands, since it is possible to red shift the $660 \mathrm{~nm}$ band to $674.5 \mathrm{~nm}$ by the use of different solvents. But this mechanism cannot account for chlorophylls absorbing at wavelengths of $683 \mathrm{~nm}$ and above.

The most widely accepted model, based on the interpretation of the bacteriochlorophyllprotein data by PHILIPSON and SAUER (23), accounts for multiple absorption bands by exciton interaction. An exciton state exists when two molecules are so close that it is not possible to excite one without exciting the other. The distance is typically $20 \dot{A}$ or less, which is less than twice the diameter of the porphyrin ring, and only 5 times its thickness (25). The interactions result in the splitting of energy levels, with a consequent splitting of the absorption spectrum. The strength of the absorption is proportional to the area under the absorption curve and is related to the dipole strength, which is not greatly affected by the exciton interaction but which has a large influence on the rotational strengths, giving rise to large positive and negative peaks in the $C D$ spectrum (25). If the chlorophyll molecules are too close $(<10 \dot{A})$, they can form an excimer, within which the excitation energy absorbed by one of them is dissipated (1). Thus chlorophyll molecules must not be randomly distributed within a chlorophyll-protein to avoid coming too close to one another, and in fact the average distance between bacteriochlorophyll molecules in the bacteriochlorophyll-protein is $12 \dot{A}(22)$.

Analysis of the absorption spectra of isolated chlorophyll-proteins $(3,4,14,15,24,28)$, and of Chlamydomonas mutants $(2,8)$ by Gaussian deconvolution and their fourth derivative, has shown that certain long wavelength absorbing forms of chlorophyll $a$ are specifically associated with PSII (Ca683) and others with PSI (Ca691). The present paper reports the low temperature absorption spectra from a number of barley nuclear gene mutants lacking one or more 
specific chlorophyll-proteins. This has involved both Gaussian deconvolution and fourth derivative spectroscopy.

\section{MATERIALS AND METHODS}

\subsection{Plant material}

Seeds of wild type and mutant barley (Hordeum vulgare L.) were germinated in tap water-moistened vermiculite and grown for 7 days at $22{ }^{\circ} \mathrm{C}$ under continuous illumination (1700 lux) from Sylvania Gro-lux fluorescent tubes.

\subsection{Spectroscopy}

Samples were prepared in several different ways for low temperature $(77 \mathrm{~K}$ ) absorption spectroscopy. Thylakoids were isolated from pre-cooled seedling leaves by grinding in 0.4 M-sucrose, $50 \mathrm{~mm}$-Tricine, pH 7.9 and $5 \mathrm{~mm}$ $\mathrm{MgCl}_{2}$ and were centrifuged for $5 \mathrm{~min}$ at $1500 \times \mathrm{g}$. The re-suspended thylakoids were then adsorbed onto the surface of a millipore filter to a concentration corresponding to about $0.3 \mathrm{~A}$ at $675 \mathrm{~nm}$, and then used for spectroscopy, as described by BENNOUN and JUPIN (2). Alternatively, chloroplasts were extracted from leaves by scraping with a razor blade in the presence of grinding buffer, and the expressate was made $60 \%$ with respect to glycerol, and frozen as a clear glass onto clear acrylic film. The most rapid method, which gave equally good spectra, involved attaching seedling leaves to transparent adhesive tape and carefully abrading away cell layers with an injector type razor blade until the absorption of the leaf at $675 \mathrm{~nm}$ was reduced to about $0.3 \mathrm{~A}$. The tape was then applied over the sample slit of a specially constructed metal specimen holder, and plunged into a Dewar vessel containing liquid nitrogen. Absorption spectra at $77 \mathrm{~K}$ were made with an Aminco DW2a spectrophotometer using the liquid nitrogen attachment. The reference slit contained transparent adhesive tape, and the baseline was corrected beforehand. Spectra were recorded from $400-800 \mathrm{~nm}$ at a scan rate of $5 \mathrm{~nm} \cdot \mathrm{s}^{-1}$ and a slit width of $3 \mathrm{~nm}$, and recorded on graph paper and simultaneously on a Nicolet Explorer III digital oscilloscope and stored on disc. Data was transferred to a Hewlett-Packard 9836S desktop computer for subsequent analysis.

\subsection{Fourth derivative spectra}

The fourth derivative of the absorption spectra was calculated according to the method of BUTLER and HOPKINS (6), which involves the use of slightly different intervals for successive differentiations to obtain an optimal signal-tonoise ratio. Values of $3.0,3.2,3.6$ and $3.8 \mathrm{~nm}$ were used routinely, the difference between these figures having the 1:2:1 ratio recommended by BUTLER and HOPKINS (6). The calculations were carried out over the range 600-725 nm, with data points every $0.1 \mathrm{~nm}$, and plotted as a travelling average over an interval of $1.6 \mathrm{~nm}$. The fourth derivative of the sum of the Gaussian fit was either calculated over a fixed interval of nominally $3 \mathrm{~nm}$, or from the mathematical formula for a Gaussian curve (7):

$$
\begin{aligned}
A & =A_{0} e^{-c x^{2}} \\
A^{\mathrm{IV}} & =\frac{4 A_{0} c^{2}}{\omega^{4}}\left(4 c^{2} x^{4}-12 c x^{2}+3\right) e^{-c x^{2}}
\end{aligned}
$$

where:

$\mathrm{A}_{\mathrm{o}}=$ peak height

$c=4 \ln 2$

$x=\frac{\lambda-\lambda_{0}}{\omega}$

$\omega=$ bandwidth at $A=1 / 2 A_{0}$

$\lambda_{0}=$ wavelength maximum

\subsection{Gaussian deconvolution}

The original data was averaged to reduce noise, and one point every $0.5 \mathrm{~nm}$ between 600 and $725 \mathrm{~nm}$ ( $=250$ points) was used for analysis by Gaussian deconvolution. The programme, which is based on a non-linear least-squares best fit, is a BASIC translation of the FORTRAN programme RESOL of Glenn Ford, Carnegie Institute, Washington. The initial estimates for the parameters of 10 pure Gaussian curves are entered manually, and the programme evaluates the goodness-of-fit $(\sigma)$ for every tenth point until the residual sum of squares is reduced to less than 1 , and subsequent iterations are made for each of the 250 points. The difference between the original data and the sum of the Gaussians is expanded by a scale factor and plotted as an error curve below the absorption spectrum. 

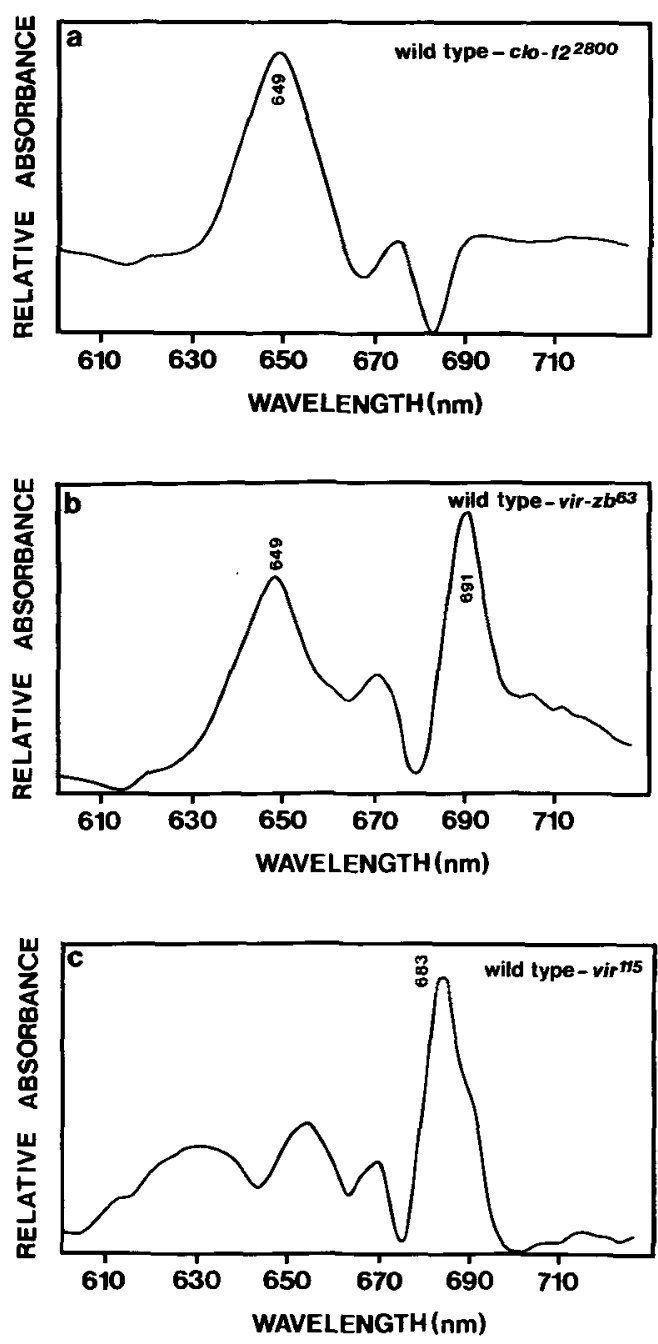

Figure 2. Low temperature $(77 \mathrm{~K})$ difference spectra of wild type and mutant barley, showing the loss of specific components from the mutants. Scales factors for re-normalisation of the spectra are (a) 2.57 (b) 5.82 and (c) 4.02, and yield amplitudes for the respective peaks at 649,691 and $683 \mathrm{~nm}$ of 312,163 and 249 .

\section{RESULTS}

\subsection{Difference absorption spectra}

Several different absorption peaks could be seen as shoulders on the main chlorophyll $Q_{y}$ absorption band of wild type barley seedling leaves. Specific peaks were detected by difference spectra of wild type and mutant leaves (Fig. 2). After subtraction, spectra were re-nor-

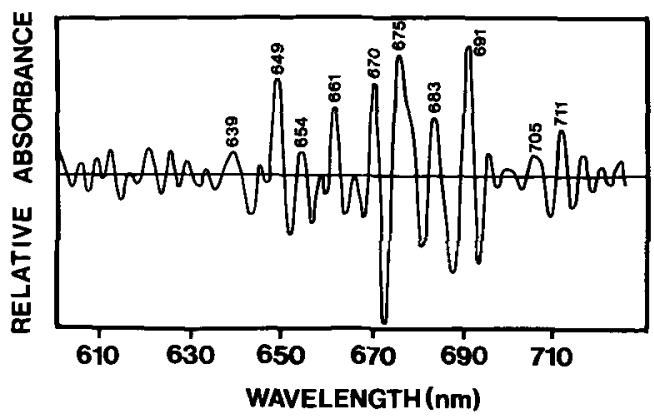

Figure 3. Fourth derivative of wild type thylakoids using a nominal differentiating interval (dx) of $1.6 \mathrm{~nm}$. Apart from the major peaks at $639,649,661,670,675$, 683 and $691 \mathrm{~nm}$, minor peaks are also found at 654 , 705 and $711 \mathrm{~nm}$, with a shoulder at $678 \mathrm{~nm}$. These minor peaks are not present in all preparations, and are often only just above the noise level.

malised so that maximum and minimum values were 0 and 1000 , respectively. Thus the chlorophyll $b$-less mutant $c l o-f 2^{2800}$ lacks the chlorophyll $b$ absorption band at $649 \mathrm{~nm}$ (Fig. 2a), while the PSI-less mutant vir-z $b^{63}$ lacks a chlorophyll $a$ species absorbing at $691 \mathrm{~nm}$ (Fig. 2b), and the PSII-less mutant vir- ${ }^{115}$ lacks chlorophyll $a$ absorbing at $683 \mathrm{~nm}$ (Fig. 2c). The peak heights and scale factors used for re-normalisation allow the calculation of the amplitudes $\left(A_{0}\right)$ of these components in the wild type absorption spectrum. Thus an amplitude of 312 is predicted for the peak at $649 \mathrm{~nm}, 163$ for the $691 \mathrm{~nm}$ peak and 249 for the $683 \mathrm{~nm}$ component (cf Table II).

\subsection{Wild type}

The low temperature absorption spectrum of wild type seedlings was independent of preparation technique. The fourth derivative of the spectrum from $600-725 \mathrm{~nm}$ over an interval of $3 \mathrm{~nm}$ (Fig. 4b) resolved distinct peaks at 639 , $648,661,669,677,683$ and $691 \mathrm{~nm}$. These values were used as the initial estimates of the wavelength maxima $\left(\lambda_{0}\right)$ for 7 symmetric pure Gaussian components, with $\omega=10 \mathrm{~nm}$, plus three others at 589, 626 and $698 \mathrm{~nm}$ with broader widths. It was possible, however, to resolve further peaks in the fourth derivative by using a shorter differentiating interval, although 

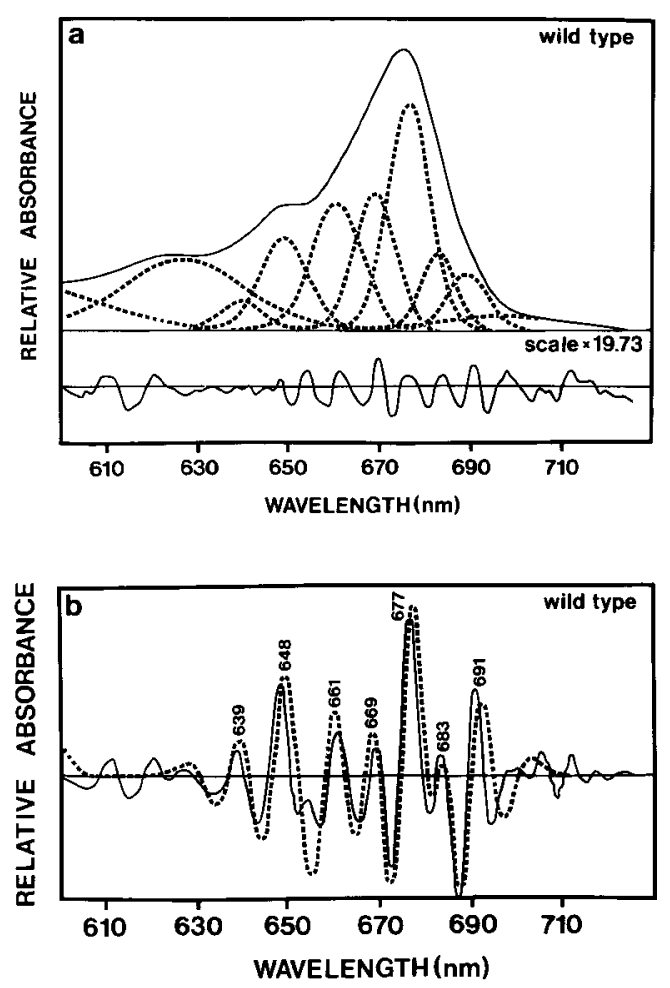

Figure 4. (a) Low temperature $(77 \mathrm{~K})$ absorption spectrum of wild type barley showing the solution provided by Gaussian deconvolution. In addition to the 7 narrow Gaussian components, 3 broad components at $589(\omega=43 \mathrm{~nm}), 626(\omega=32 \mathrm{~nm})$ and $698 \mathrm{~nm}$ $(\omega=31 \mathrm{~nm})$ are present.

(b) Fourth derivative of the absorption spectrum (-) and of the sum of the Gaussian components (---), both at $\mathrm{dx}=3 \mathrm{~nm}$. The match is very close, indicating that the solution is a good one.

this raised the noise level. With a nominal differentiating interval of $1.6 \mathrm{~nm}$, peaks were resolved at $639,649,654,661,670,677,683$ and $691 \mathrm{~nm}$, with peaks also seen at $666,696,705$ and $711 \mathrm{~nm}$ in some, but not all preparations (Fig. 3). In some samples, the $677 \mathrm{~nm}$ peak was split into peaks at 675 and $678 \mathrm{~nm}$, and the 661 $\mathrm{nm}$ peak could sometimes be resolved into one at $658 \mathrm{~nm}$ and a second at $662 \mathrm{~nm}$.

Since the computer-derived solution for fitting Gaussian curves is not unique, and depends largely on the choice of initial parameter values, other criteria in addition to the error sum of squares $(\sigma)$ were used. The fourth derivative of the sum of the Gaussian components should match as closely as possible that of the absorption spectrum itself (Fig. 4b) and the same Gaussian components should be present in different mutants, although with different amplitudes $\left(\mathrm{A}_{0}\right)$. In this way, the solution shown in Figure 4a and Table I was obtained. Each curve corresponds to a chlorophyll species absorbing at a specific wavelength, and the area under each curve is proportional to the number of molecules of chlorophylls in each species, assuming that all $Q_{y}$ transitions have the same dipole strength. These values have been expressed as a percentage of the 7 components resolved by fourth derivative spectroscopy (Table I).

\subsection{Chlorina- $\boldsymbol{f}^{2800}$}

The $77 \mathrm{~K}$ absorption spectrum of seedling leaves of this mutant, which lacks chlorophyll $b$, was missing the prominent peak at $650 \mathrm{~nm}$ (Fig. 5a). The fourth derivative showed the absence of the peak at $639 \mathrm{~nm}$, while those at 648 and 677 $\mathrm{nm}$ were much reduced (Fig. $5 \mathrm{~b}$ ). The best-fit by Gaussian deconvolution is shown in Figure 5a and closely matches that of the data. Compared with wild type, the component at $640.5 \mathrm{~nm}$ was missing, while that at $649.5 \mathrm{~nm}$ was shifted to $653.2 \mathrm{~nm}$, with a much reduced amplitude. The peak at $682.9 \mathrm{~nm}$ was narrower than in the wild type ( 8.2 vs $8.7 \mathrm{~nm}$ ), which accounted for the increased amplitude in the fourth derivative.

\subsection{Viridis- $z b^{63}$}

This mutant, which completely lacks the reaction centre chlorophyll-protein of PSI $(20,27)$, produced the absorption spectrum shown in Figure 6a. There was a marked reduction in the amplitude of the chlorophyll $b$ peak at $650 \mathrm{~nm}$, as confirmed by the fourth derivative, which also lacked the component at $691 \mathrm{~nm}$ (Fig. 6b), and the difference spectrum (Fig. 2b). The solution obtained by deconvolution (Fig. 6a and Table I) was consistent with these properties, with a loss of the $689 \mathrm{~nm}$ peak, attributable to the loss of the $\mathrm{P} 700 \mathrm{Chl}_{a}-\mathrm{P} 1$ (CPI), and a reduction in the amount of the $650 \mathrm{~nm}$ component, which is due 
D.J. SIMPSON: Absorption spectroscopy of barley mutants

Table I. Chlorophyll components found by Gaussian deconvolution of absorption spectra

\begin{tabular}{|c|c|c|c|c|c|c|c|}
\hline \multicolumn{8}{|c|}{ wild type $(\sigma=2.02)$} \\
\hline wavelength (nm) & 640.5 & 649.9 & 660.9 & 668.9 & 676.6 & 682.9 & 688.9 \\
\hline half-width (nm) & 10.3 & 12.0 & 12.7 & 10.4 & 10.8 & 8.7 & 10.4 \\
\hline amplitude & 101 & 327 & 447 & 486 & 798 & 268 & 193 \\
\hline area $(\%)$ & 3.6 & 13.8 & 19.9 & 17.6 & 30.0 & 8.1 & 7.0 \\
\hline \multicolumn{8}{|l|}{$\operatorname{clo}-2^{2800}(\sigma=1.45)$} \\
\hline wavelength (nm) & 640.5 & 653.2 & 661.9 & 668.9 & 676.8 & 682.8 & 688.5 \\
\hline half-width (nm) & - & 11.8 & 11.5 & 10.1 & 11.1 & 8.2 & 10.3 \\
\hline amplitude & 0 & 76 & 384 & 518 & 814 & 328 & 196 \\
\hline area $(\%)$ & 0 & 3.7 & 18.2 & 21.5 & 37.3 & 11.0 & 8.3 \\
\hline \multicolumn{8}{|l|}{$v i r-z b^{63}(\sigma=2.46)$} \\
\hline wavelength (nm) & 640.6 & 650.3 & 661.3 & 668.9 & 676.7 & 683.1 & 688.9 \\
\hline half-width (nm) & 11.3 & 12.3 & 12.0 & 10.0 & 10.5 & 8.9 & - \\
\hline amplitude & 76 & 234 & 406 & 462 & 784 & 269 & 0 \\
\hline $\operatorname{area}(\%)$ & 3.6 & 12.0 & 20.4 & 19.4 & 34.5 & 10.1 & 0 \\
\hline \multicolumn{8}{|c|}{ vir-z $b^{63} \times$ clo- $f 2^{2800}(\sigma=1.41)$} \\
\hline wavelength (nm) & 640.5 & 653.9 & 662.1 & 669.0 & 676.9 & 682.7 & 686.4 \\
\hline half-width (nm) & - & 13.8 & 11.8 & 10.1 & 10.4 & 8.0 & 12.6 \\
\hline amplitude & $\mathbf{0}$ & 84 & 376 & 507 & 805 & 274 & 124 \\
\hline area $(\%)$ & 0 & 5.1 & 19.5 & 22.3 & 36.7 & 9.6 & 6.8 \\
\hline \multicolumn{8}{|l|}{$v_{i r-}{ }^{H S}(\sigma=1.54)$} \\
\hline wavelength (nm) & 640.0 & 649.4 & 661.7 & 669.5 & 677.0 & 682.9 & 687.4 \\
\hline half-width (nm) & 11.5 & 13.1 & 12.4 & 11.2 & 11.0 & - & 13.7 \\
\hline amplitude & 104 & 323 & 386 & 427 & 701 & 0 & 209 \\
\hline $\operatorname{area}(\%)$ & 4.7 & 16.6 & 18.7 & 18.6 & 30.1 & 0 & 11.3 \\
\hline \multicolumn{8}{|c|}{$v i r-115 \times \operatorname{clo}-f 2^{2800}(\sigma=2.62)$} \\
\hline wavelength (nm) & 640.5 & 652.1 & 660.8 & 669.0 & 677.3 & 682.9 & 686.8 \\
\hline half-width (nm) & - & 15.6 & 12.6 & 12.6 & 12.6 & - & 12.1 \\
\hline amplitude & 0 & 91 & 345 & 525 & 804 & $\mathbf{0}$ & 270 \\
\hline area $(\%)$ & 0 & 5.5 & 17.0 & 25.7 & 39.1 & 0 & 12.7 \\
\hline \multicolumn{8}{|l|}{ vir-m $m^{29}(\sigma=1.51)$} \\
\hline wavelength (nm) & 640.9 & 648.7 & 659.8 & 669.1 & 676.7 & 682.8 & 686.8 \\
\hline half-width (nm) & 12.6 & 10.3 & 13.6 & 11.3 & 10.3 & 7.5 & 12.1 \\
\hline amplitude & 168 & 282 & 440 & 544 & 759 & 97 & 134 \\
\hline area $(\%)$ & 7.7 & 11.0 & 21.8 & 22.4 & 28.6 & 2.6 & 5.9 \\
\hline \multicolumn{8}{|l|}{$v i r-k^{23}(\sigma=1.67)$} \\
\hline wavelength (nm) & 640.4 & 650.6 & 662.2 & 669.8 & 676.4 & 682.9 & 690.3 \\
\hline half-width (nm) & 13.1 & 12.0 & 12.4 & 9.8 & 9.0 & 8.5 & 8.5 \\
\hline amplitude & 89 & 210 & 446 & 593 & 686 & 504 & 132 \\
\hline area $(\%)$ & 4.4 & 9.5 & 20.8 & 21.8 & 23.2 & 16.1 & 4.2 \\
\hline
\end{tabular}

to a partial deficiency of chlorophyll $b$ in this mutant, as shown by the increased chlorophyll $a / b$ ratio (5.3 versus 3.2 for the wild type). The fourth derivative of the Gaussian fit did not, however, match that of the data as closely as for other mutants (Fig. 6b). The absorption spec- trum of the double mutant, $v i r-z b^{63} \times c l o-f 2^{2800}$, is shown in Figure 7a. The fourth derivative of the Gaussian solution matches that of the absorption spectra very closely (Fig. 7b), although it required the presence of a component at 686.4 $\mathrm{nm}(\omega=12.6 \mathrm{~nm})$, which cannot be the same as 

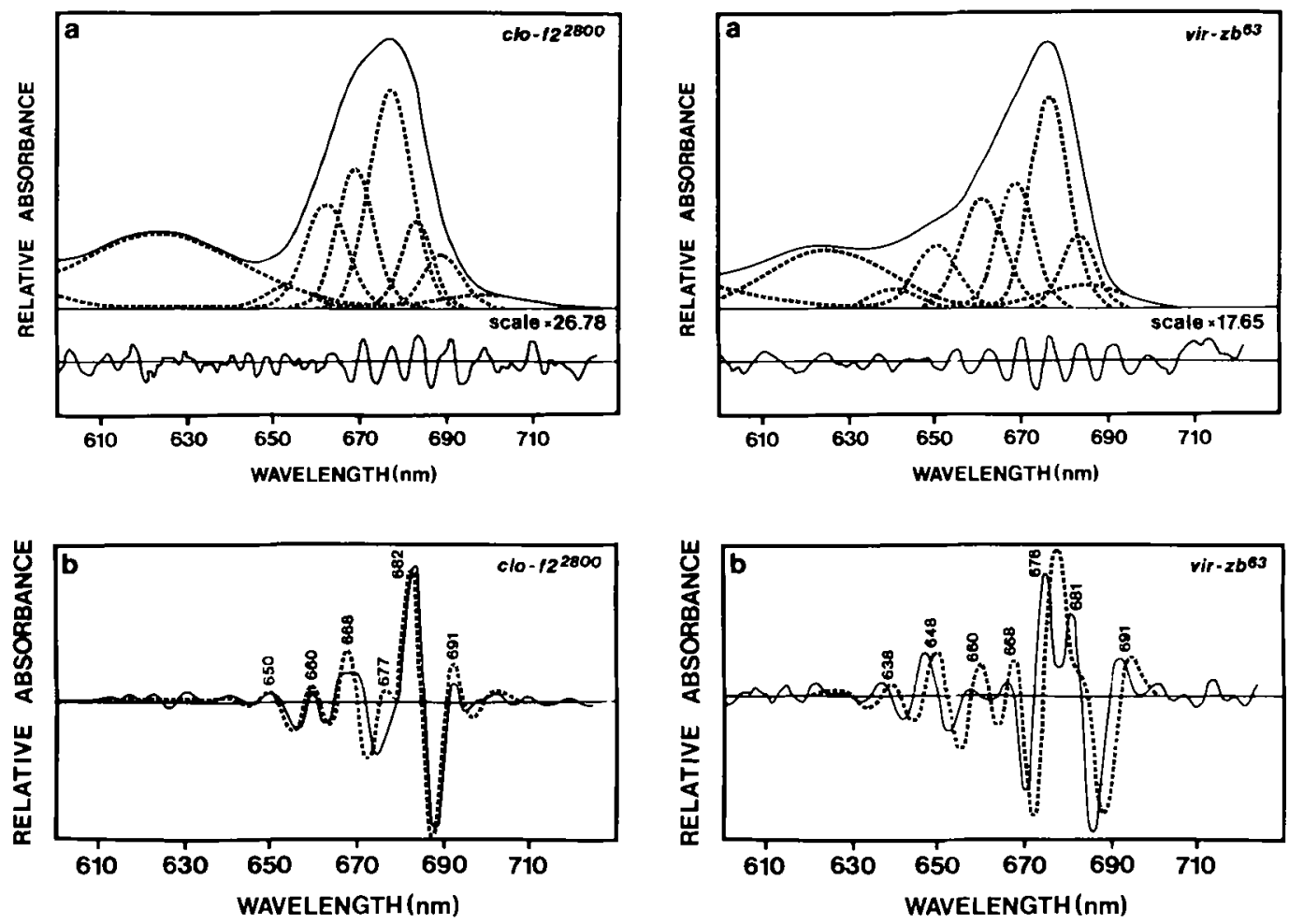

Figure 5. (a) Low temperature ( $77 \mathrm{~K})$ absorption spectrum of $c l o-f 2^{2800}$ with the solution from Gaussian deconvolution. The $640 \mathrm{~nm}$ component is missing, with a minor chlorophyll $a$ component required at 653 $\mathrm{nm}$ in the absence of the $650 \mathrm{~nm}$ peak.

(b) Fourth derivative of the absorption spectrum (-) and Gaussian solution (--) at $\mathrm{dx}=3 \mathrm{~nm}$.

the $689.9 \mathrm{~nm}$ component, and which was not found in wild type (Table I). The absorption spectrum of this double mutant can be attributed to the PSII core, plus LHCI and CP29, the latter two being stable in the absence of chlorophyll $b$ (13).

\subsection{Viridis- ${ }^{115}$}

The thylakoids of viridis- ${ }^{115}$ have recently been shown to lack completely the PSII reaction centre (26). This did not result in a major change in the absorption spectrum (Fig. 8a), but the absence of a component at $683 \mathrm{~nm}$ was clearly demonstrated by taking the fourth derivative

Figure 6. (a) Low temperature (77 K) absorption spectrum of $v i r-z b^{63}$ with the solution from Gaussian deconvolution. Correlated with the loss of PSI activity is the absence of the $689 \mathrm{~nm}$ component.

(b) Fourth derivative of the absorption spectrum (-) and Gaussian solution (---) at $\mathrm{dx}=3 \mathrm{~nm}$. The match is not as good as for other mutants and indicates that some of the components may be a mixture of Gaussian and Lorentzian curves.

(Fig. 8b). This component was also missing from the solution obtained by Gaussian deconvolution (Fig. 8a and Table I), and was clearly seen in the difference absorption spectrum (Fig. 2c). The double mutant vir- ${ }^{115} \times$ clo- $f 2^{2800}$, lacked those components which were missing in each of the parents, i.e., $640.5 \mathrm{~nm}, 649.9 \mathrm{~nm}$ and $682.9 \mathrm{~nm}$ (Fig. 9 and Table I). The remaining components must correspond, in view of the loss of PSII and most of the chlorophyll $a / b$ proteins, to PSI, consisting of $\mathrm{P700} \mathrm{Chl}_{a}-\mathrm{Pl}$ plus LHCI and CP29. 

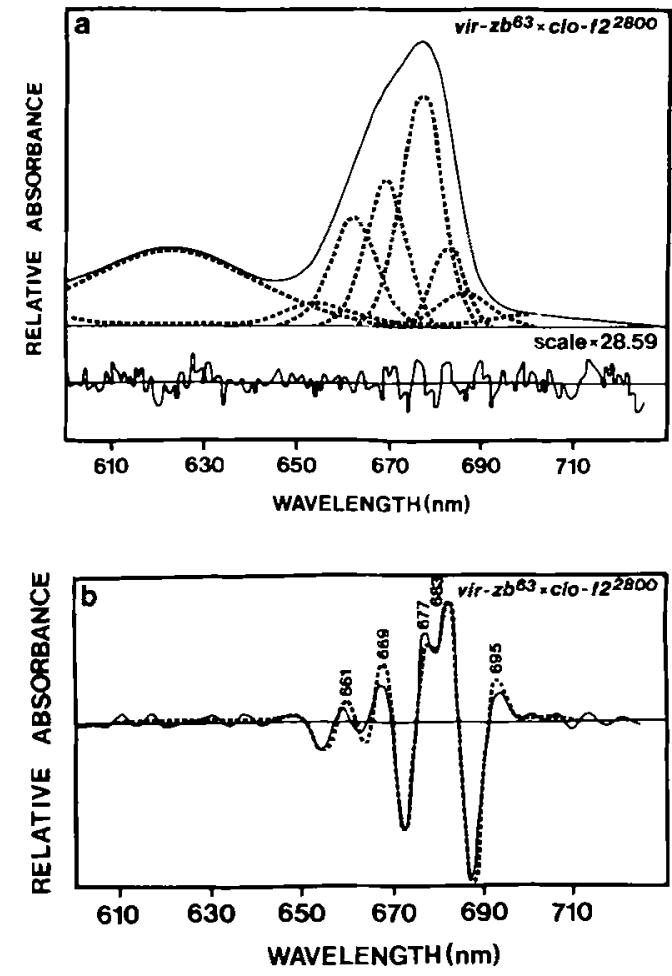

Figure 7. (a) Low temperature (77 K) absorption spectrum of the double mutant $v i r-z b^{63} \times c l o-f 2^{2800}$ with the solution from Gaussian deconvolution. This mutant should contain the PSII core, plus LHCI containing only chlorophyll $a$. Bands at 640,650 and $689 \mathrm{~nm}$ are missing.

(b) Fourth derivative of the absorption spectrum (-) and Gaussian solution (---) at $\mathrm{dx}=3 \mathrm{~nm}$. The two curves match very closely.

\subsection{Viridis $-m^{29}$}

This mutant contains only about $25 \%$ of the PSII reaction centres that are found in wild type (27). The fourth derivative of the absorption spectrum was unable to resolve a peak at 683 $\mathrm{nm}$, although a shoulder was present (Fig. 10b). Gaussian deconvolution showed a peak at 683 nm (Fig. 10a and Table I), but its area was only about $1 / 3$ that of wild type (Table I).

\subsection{Viridis- $k^{23}$}

This mutant is photosynthetically competent $(21,27)$, but is deficient in chlorophyll $b$ and
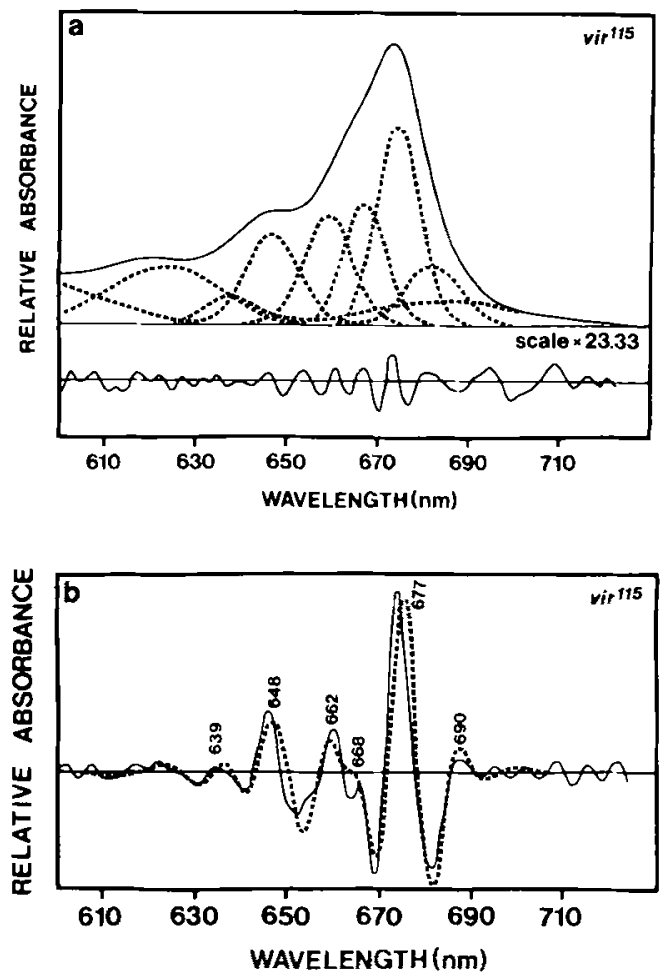

Figure 8. (a) Low temperature $(77 \mathrm{~K}$ ) absorption spectrum of vir-"1's with the solution from Gaussian deconvolution. Correlated with the loss of PSII activity is the absence of the $683 \mathrm{~nm}$ component.

(b) Fourth derivative of the absorption spectrum (-) and Gaussian solution (-- ) at $\mathrm{dx}=3 \mathrm{~nm}$.

lacks LHCI (13), so its absorption spectrum was expected to reflect that of the reaction centres of PSI and PSII lacking most or all of their lightharvesting antennae (Fig. 11a). The fourth derivative showed a prominant peak at $683 \mathrm{~nm}$, but all peaks found in wild type were also present, indicating that $\mathrm{LHCI}$ contains no specific chlorophyll $a$ species. The fourth derivative of the solution from Gaussian deconvolution (Table I), which closely matched that previously published (21), was close to that of the original spectrum (Fig. 1 lb).

\section{DISCUSSION}

It has been possible to deconvolute the $77 \mathrm{~K}$ absorption spectra of wild type and mutant 

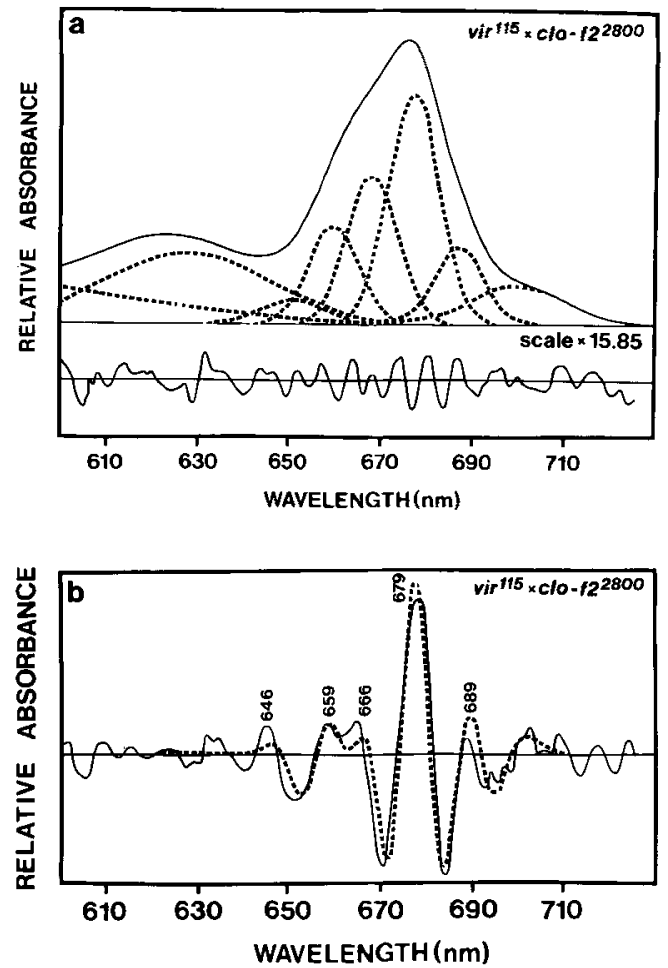

Figure 9. (a) Low temperature $(77 \mathrm{~K})$ absorption spectrum of the double mutant vir- ${ }^{115} \times$ clo-f $2^{2800}$ with the solution from Gaussian deconvolution. Bands at 640,650 and $683 \mathrm{~nm}$ are missing. This mutant should contain PSI plus LHCI containing only chlorophyll $a$. (b) Fourth derivative of the absorption spectrum (-) and Gaussian solution (---) at $\mathrm{dx}=3 \mathrm{~nm}$.

barley leaves using the same 7 symmetric Gaussian components. The fourth derivative of the solution is usually very similar to that of the original spectrum, and consistent with difference absorption spectra, suggesting that the mathematically derived components represent real chlorophyll species in vivo. By lowering the temperature, from $293 \mathrm{~K}$ to $77 \mathrm{~K}$, the absorption bandwidths decrease due to reduced molecular vibration and rotation, allowing improved resolution. The appearance of additional bands in vivo at $77 \mathrm{~K}$ is attributed to modification of the excitonic coupling interactions between chlorophyll molecules, causing band splitting or band shifting towards higher or lower wavelengths as a result of changes in the intermolecular dis-
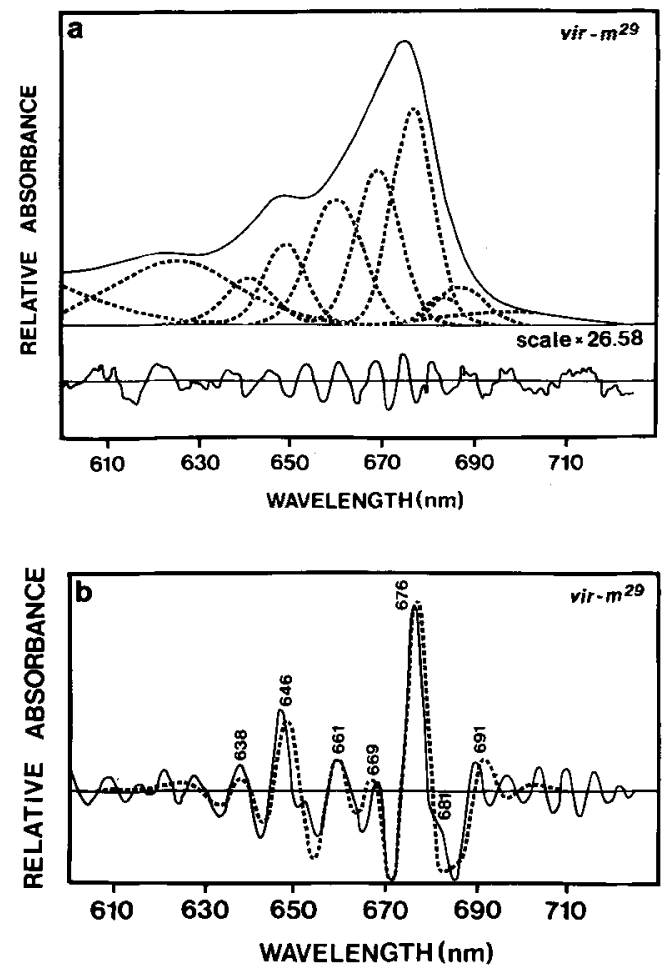

Figure 10. (a) Low temperature (77 K) absorption spectrum of vir- $m^{29}$ with the solution from Gaussian deconvolution. Correlated with the partial loss of PSII activity is a corresponding reduction in the amplitude of the $683 \mathrm{~nm}$ component.

(b) Fourth derivative of the absorption spectrum (-) and Gaussian solution (---) at $\mathrm{dx}=3 \mathrm{~nm}$.

tances or orientation between chlorophyll molecules, and is not a low temperature artefact (15). No changes are seen at low temperature in the $650-670 \mathrm{~nm}$ region, as expected from the low degree of organisation of the monomeric chlorophylls absorbing in this region (15).

The fourth derivative spectrum of wild type barley leaves closely resembled those of spinach (7), tobacco $(15,24)$, pea (4), and that of barley reported by (5). The method of calculating the fourth derivative described by (7), using four nearly but not exactly equal differentiating intervals, greatly reduced the amount of noise. But it was not possible to resolve reproducibly components at wavelengths greater than $691 \mathrm{~nm}$, where the absorption is weak, because of the low 

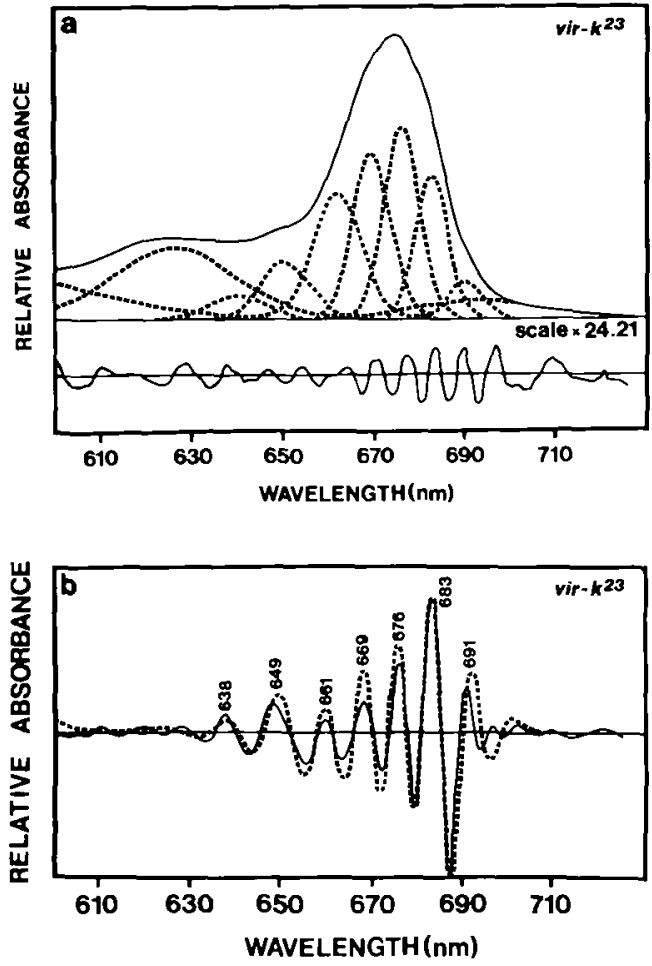

Figure 11. (a) Low temperature ( $77 \mathrm{~K}$ ) absorption spectrum of vir- $k^{23}$ with the solution from Gaussian deconvolution. This mutant lacks $\mathrm{LHCI}$ and most of the LHCII, but no bands are missing, although their amplitudes are significantly different from that of wild type.

(b) Fourth derivative of the absorption spectrum (-) and Gaussian solution (---) at $\mathrm{dx}=3 \mathrm{~nm}$.

signal-to-noise ratio (16). Although only 7 peaks were used for subsequent curve-fitting, there is evidence for the existence of other components when the differentiating interval is reduced (Fig. 3 , refs. 5, 24).

Two Gaussian curves can be resolved when they are separated by more than half the band width $(\omega / 2)$ of the narrower curve (Fig. 12). In contrast, two Lorentzian curves (6) can be resolved when they are separated by only $\omega / 4$ (Fig. 13). Thus the resolution of peaks at 675 and 678 $\mathrm{nm}$ or 658 and $662 \mathrm{~nm}$, where $\omega=10.8$ or 12.7 nm (Table I), implies that the components are not pure Gaussians, as also concluded by LECLERC et al. (16) and BROWN and SCHOCH (4).
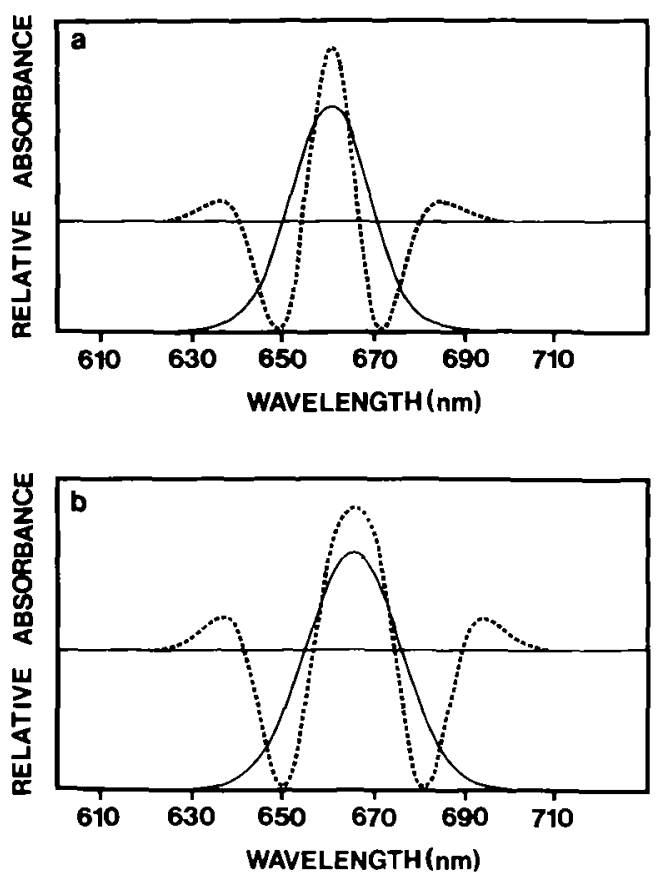

Figure 12. (a) A Gaussian curve with $\lambda=660 \mathrm{~nm}$, $\omega=20 \mathrm{~nm}, \mathrm{~A}_{0}=1000(-)$ and its fourth derivative $(---)$.

(b) Two Gaussian curves with $\lambda_{1}=660 \mathrm{~nm}, \lambda_{2}=670$ $\mathrm{nm}, \omega=20 \mathrm{~nm}$ and $\mathrm{A}_{0}=500$ (一). They are not resolved by the fourth derivative (---).

To resolve two curves separated by $\omega / 3$, each must be at least 10-20\% Lorentzian. Since pure Gaussians were used exclusively in the curve-fitting, this may explain some of the discrepancies between the fourth derivatives of the fitted solution and the original spectrum (e.g., Fig. 6b).

The complexity of the absorption spectrum, which arises from the large number of chlorophyll molecules and their different associations in vivo, makes it difficult to analyse. Thus, fourth derivative spectra are mainly used to determine the number and position of close absorption bands (6), within the limits of resolution imposed by the technique. Although the bandwidth of the fourth derivative peaks are proportional to the bandwidths $(\omega)$ of the components in the original spectrum, in practice it is difficult to measure these values since the peaks are often too close together. However, since the amplitude of the fourth derivative is proportion- 

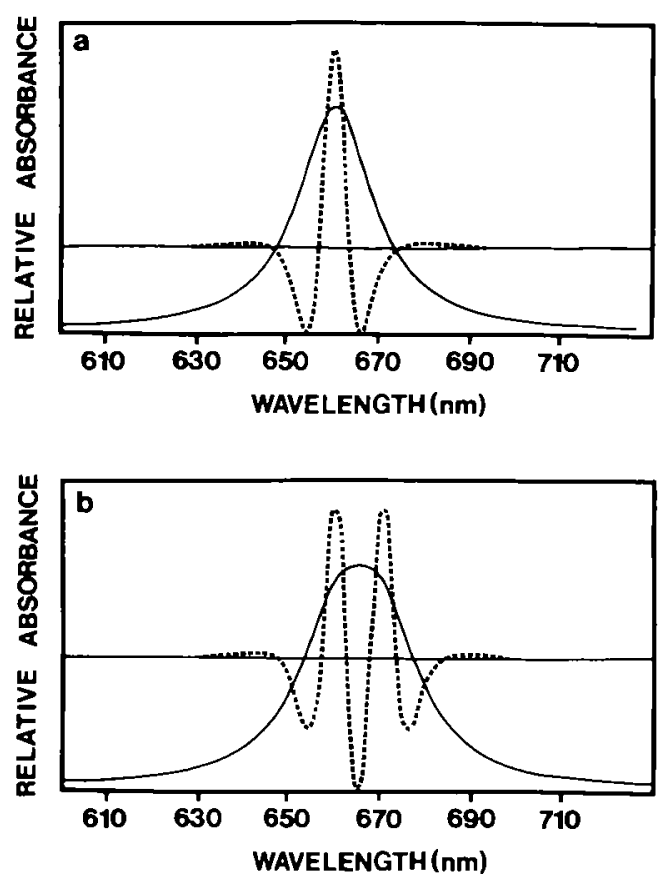

Figure 13. (a) A Lorentzian curve with $\lambda=660 \mathrm{~nm}, \omega$ $=20 \mathrm{~nm}, A_{\mathrm{o}}=1000(-)$ and its fourth derivative (-- ).

(b) Two Lorentzian curves with $\lambda_{1}=660 \mathrm{~nm}, \lambda_{2}=670$ $\mathrm{nm}, \omega=20 \mathrm{~nm}$ and $A_{0}=500$ (-). They are readily resolved by the fourth derivative (---).

al to $1 / \omega^{4}$ for both Gaussian and Lorentzian curves (6), the method is very sensitive to minor components with narrow bandwidths. In the present paper, the fourth derivative has also been used to test the various different solutions resulting from Gaussian deconvolution.

The individual chlorophyll-protein complexes in thylakoids contain many different chlorophyll species, but it is possible to assign certain absorption bands in the total spectrum to specific chlorophyll-proteins. This can be done by isolating the chlorophyll-proteins $(3,14,24$, 28 ), although the use of detergents during the isolation is a possible source of artefact. Alternatively, specific chlorophyll-proteins can be removed through mutation, and the absorption spectra of the resulting mutants recorded in vivo. These spectra are still very complex, but difference spectra and fourth derivative analysis show the loss of specific absorption bands as the result of the loss of certain chlorophyll-proteins.

In the mutant clo- $f 2^{2800}$, the loss of all chlorophyll $b$ results in the complete loss of the 640.5 $\mathrm{nm}$ component, and another at $649.9 \mathrm{~nm}$, as revealed by deconvolution (Tables I and II) and the fourth derivative (Fig. 5b), although the $640.5 \mathrm{~nm}$ component is not seen in the difference spectrum (Fig. 2a). It is unlikely that the $640 \mathrm{~nm}$ component is due to a chlorophyll $a$ species, nor is it associated with the $682 \mathrm{~nm}$ band, as suggested by LEPPINK and THOMAS (17), since it is also found in vir- ${ }^{\prime \prime}$, which completely lacks the $683 \mathrm{~nm}$ component. The loss of the $649.9 \mathrm{~nm}$ band reveals a $653.2 \mathrm{~nm}$ component by Gaussian deconvolution, but this is not a prominant peak in the fourth derivative of

Table II. Normalised areas of Gaussians

\begin{tabular}{|c|c|c|c|c|c|c|c|c|c|c|c|}
\hline Component (nm) & 640.5 & 649.9 & 653 & 660.7 & 668.9 & 676.6 & 682.9 & 688.9 & chl $a$ & chl $b$ & $\operatorname{chl} a / b$ \\
\hline wild type* & 30.6 & 89.4 & 14.0 & 88.1 & 78.0 & 133 & 36.0 & 31.1 & 380 & 120 & 3.17 \\
\hline $\operatorname{clo} f 2^{2800^{\circ}}$ & 0 & 0 & 14.0 & 68.3 & 80.8 & 140 & 41.2 & 31.3 & 376 & 0 & $\infty$ \\
\hline vir-z $z b^{638}$ & 22.2 & 44.1 & 15.9 & 66.3 & 62.9 & 112 & 32.6 & 0 & 290 & 66 & 4.4 \\
\hline vir-z $b^{63} \times \operatorname{clo-f2^{28005}}$ & 0 & 0 & 15.9 & 60.5 & 69.3 & 114 & 29.8 & 0 & 290 & 0 & $\infty$ \\
\hline vir-115*" & 35.4 & 98.5 & 14.0 & 74.3 & 73.9 & 119 & 0 & 44.5 & 326 & 134 & 2.43 \\
\hline$v_{i r-H}{ }^{H S} \times \operatorname{clo}-f^{2800 \Delta}$ & 0 & 0 & 16.7 & 51.6 & 78.1 & 119 & 0 & 38.6 & 304 & 0 & $\infty$ \\
\hline vir-m $m^{200}$ & 62.2 & 47.4 & 21.9 & 92.5 & 95.1 & 121 & 11.3 & 25.0 & 367 & 110 & 3.34 \\
\hline$v i r-k^{23+}$ & 29.9 & 28.1 & 19.0 & 74.1 & 78.0 & 81.1 & 57.7 & 15.0 & 244 & 58 & 5.5 \\
\hline
\end{tabular}

* normalisation factors: $\mathrm{Chl} a=380 / 26135, \mathrm{Chl} b=120 / 4338$

5 all values re-normalised to account for the loss of 90 molecules of $\mathrm{Chl} a$ as CPI

$\Delta$ all values re-normalised to give same number of molecules of $\mathrm{Ca} 677$ as vir-"

+ all values re-normalised to give same number of molecules of Ca669 as wild type 
clo $f 2^{2800}$ or any of the double mutants (Figs. $5 \mathrm{~b}$, $7 \mathrm{~b}$ and $9 \mathrm{~b})$. This may be the chlorophyll $a$ species giving rise to the $654 \mathrm{~nm}$ peak in the wild type fourth derivative at high resolution (Fig. 3), although its presence does not improve the goodness-of-fit of the deconvolution of the wild type spectrum.

In Table II, the areas of the various components have been normalised for wild type, so that they are expressed per 500 chlorophyll molecules, assuming equal dipole strengths for all chlorophyll $a$ components. For the mutants, the normalisation has been made using the same conversion factors for chlorophyll $a$ and $b$, with further normalisation as explained in Table II, although these values are not meant to be strictly quantitative. There is nevertheless, a clear loss of chlorophyll $a$ absorbing at $661 \mathrm{~nm}$ from clo- $f 2^{2800}$ which is probably associated with the loss of most of the LHCII.

The $689 \mathrm{~nm}$ component is clearly associated with about 30 molecules of chlorophyll $a$ in the PSI reaction centre, since this band is lost from the PSI-deficient mutant vir-z $b^{63}$ (Table II). This is consistent with Chlamydomonas, where BENNOUN and JUPIN (2) concluded that about 46/ 500 molecules of chlorophyll $a$ were associated with a PSI-specific band absorbing at $691 \mathrm{~nm}$. Although this component has been found in isolated CPI $(24)$ and PSI preparations $(3,4)$, it was not enriched compared to whole thylakoids, which is surprising since the chlorophyll:P700 ratios of these preparations are 90:1 and 200:1, compared with about $600: 1$ for thylakoids.

The mutant vir- ${ }^{115}$ establishes that the 30-40 molecules of chlorophyll $a$ absorbing at $683 \mathrm{~nm}$ in wild type thylakoids (Table II) are specifically associated with the PSII reaction centre, since both PSII activity and the $683 \mathrm{~nm}$ band are missing from this mutant. The reduced intensity of the $683 \mathrm{~nm}$ band in vir- $m^{29}$ (Table II) is consistent with the partial loss of PSII activity. Also consistent with this assignment is the presence of a $683 \mathrm{~nm}$ component $(\omega=8 \mathrm{~nm})$ in PSII core particles (28). This band represents $8-10$ of the 45 chlorophyll molecules in the complex, and consists of more than one component, including the reaction centre pigment $\mathrm{P680}$ and pheophytin $a$.

By taking the fourth derivative of the solution provided by Gaussian deconvolution, it has been possible to select, from the many possible solutions, one which is consistent with the fourth derivative of the original absorption spectrum - a technique previously only used by LECLERC et al. (16). This has produced a series of consistent solutions for a number of mutants (Table I), allowing certain components to be assigned to specific chlorophyll-protein complexes. The validity of the solutions are further confirmed by the close correspondence of the amplitudes of these components (i.e., 650, 683 and $689 \mathrm{~nm}$ ) with those predicted from the difference absorption spectra. The large number of different chlorophyll $a$ species associated with each of the different chlorophyll-proteins has made it difficult to resolve extra bands in mutants lacking some chlorophyll-proteins, although a component at $653 \mathrm{~nm}$ is revealed in mutants lacking chlorophyll $b$, which absorbs at $650 \mathrm{~nm}$. Further refinement of the analysis of the absorption spectra of barley seedling leaves will require the fitting of components which have a partial Lorentzian component. The analysis is simplified, and possibly more informative, when using isolated complexes from which much of the chlorophyll has been removed, such as PSII core complexes (28), or wet etherwashed PSI reaction centres (14).

\section{ACKNOWLEDGEMENTS}

Professor DITER VON WETTSTEIN is thanked for his encouragement and support during the course of this work, and for critically reading the manuscript. I am indebted to Dr. JEANETTE BROWN of the Carnegie Institute of Washington, who provided the computer programme RESOL which was used during the course of this work. KIRSTEN KRISTIANSEN and BENTE JENSEN are acknowledged for propagating the mutant seed stocks and for producing the double mutant. I am grateful to ANN-SOFI STEINHOLTZ and NINA RASMUSSEN for their excellent work in photography and in drawing the figures. 


\section{REFERENCES}

1. Beddard, G.S. \& G. Porter: Concentration quenching in chlorophyll. Nature 260,366-367 (1976)

2. BenNoun, P. \& H. JuPIN: Spectral properties of system l-deficient mutants of Chlamydomonas reinhardi. Possible occurrence of uphill energy transfer. Biochim. Biophys. Acta 440, 122-130 (1976)

3. BROWN, J.S., J.M. ANDERSON \& L.H. GRIMME: Antenna chlorophyll a complexes in mutant and developing barley. Photosyn. Res. 3, 279-291 (1982)

4. BROWN, J.S. \& S. SCHoch: Spectral analysis of chlorophyll-protein complexes from higher plant chloroplasts. Biochim. Biophys. Acta 636, 201209 (1981)

5. BROWN, J.S. \& S. SCHOCH: Comparison of chlorophyll a spectra in wild-type and mutant barley chloroplasts grown under day or intermittent light. Photosyn. Res. 3, 19-30 (1982)

6. BUTLER, W.L. \& D.W. HoPKINS: Higher derivative analysis of complex absorption spectra. Photochem. Photobiol. 12, 439-450 (1970a)

7. Butler, W.L. \& D.W. Hopkins: An anlysis of fourth derivative spectra. Photochem. Photobiol. 12, 451-456 (1970b)

8. Delepelaire, P.: Energy transfer in the photosystem I unit of the green alga Chlamydomonas reinhardtii. Photobiochem. Photobiophys. 1, 139146 (1980)

9. FRENCH, C.S.: The distribution and action in photosynthesis of several forms of chlorophyll. Proc. Nat. Acad. Sci., USA 68, 2893-2897 (1971)

10. FRENCH, C.S.. J.S. BRown \& M.C. LaWrence: Four universal forms of chlorophyll a. Plant Physiol. 49, $421-429$ (1972)

11. KIRK, J.T.O. \& R.A.E. TILnEy-BassetT: The Plastids. Elsevier/North Holland Biomedical Press. Amsterdam, New York, London (1978)

12. HinZ, U. \& K.G. WELINDER: The light-harvesting complex of photosystem II in barley. Structure and chlorophyll organization. Carlsberg Res. Commun. 52, 39-54 (1987)

13. Høyer-Hansen, G., R. Bassi, L.S. Hønberg \& D.J. SIMPSON: Immunological characterization of chlorophyll $a / b$-binding proteins of barley thylakoids. Planta 173, 12-21 (1988)

14. IKEGAMI, I. \& S. ITOH: Chlorophyll organization in P-700-enriched particles isolated from spinach chloroplasts. CD and absorption spectroscopy. Biochim. Biophys. Acta 851, 75-85 (1986)

15. LeClerC, J.C., J. HoARAU \& R. REMY: Analysis of absorption spectra changes induced by temperature lowering of phycobilisomes, thylakoids and chlorophyll-protein complexes. Biochim. Biophys. Acta 547, 398-409 (1979)

16. Leclerc, J.C., J. Hoarau \& E. Guérin-DumarTRAIT: An analysis of Porphyridium absorption bands with a digital spectrophotometer. Photochem. Photobiol. 22, 41-48 (1975)

17. Leppink, G.J.\& J.B. Thomas: On the identity of the 640-nm component observed in chlorophyll b absorption spectra in vivo. Biochim. Biophys. Acta 305, 610-617 (1973)

18. LiTVIN, F.F.\& V.A.SINESHCHEKov: Molecular organization of chlorophyll and energetics of the initial stages in photosynthesis. In: Bioenergetics of Photosynthesis (Ed. Govindjee). Academic Press, New York, San Francisco, London. pp. 619-661 (1975)

19. LuNDFll, D.J., A.N. Glazer, A. Melis \& R. MALKIN: Characterization of a cyanobacterial photosystem I complex. J. Biol. Chem. 260, 646-654 (1985)

20. Møllf.r, B.L., R.M. SMillie \& G. Høyer-Hansen: A photosystem I mutant in barley (Hordeum vulgare L.). Carlsberg Res. Commun. 45, 87-100 (1980)

21. Nielsen, N.C., R.M. Smillie, K.W. Henningsen \& D. vON WeTtSTEIN: Composition and function of thylakoid membranes from grana-rich and granadeficient chloroplast mutants of barley. Plant Physiol. 63, 174-182 (1979)

22. Olson, J.M., B. KE \& K.H. ThOMPSON: Exciton interaction among chlorophyll molecules in bacteriochlorophyll a proteins and bacteriochlorophyll a reaction center complexes from green bacteria. Biochim. Biophys. Acta 430, 524-537 (1976)

23. PHILIPSON, K.D.\& K SAUER: Exciton interaction in a bacteriochlorophyll-protein from Chloropseudomonas ethylica. Absorption and circular dichroism at $77^{\circ} \mathrm{K}$. Biochemistry $11,1880-1885$ (1972)

24. REmy, R. J. Hoarau \& J.C. Leclerc: Electrophoretic and spectrophotometric studies of chlorophyll-protein complexes from tobacco chloroplasts. Isolation of a light harvesting pigment protein complex with a molecular weight of 70,000. Photochem. Photobiol. 26, 151-158 (1977)

25. SaUer, K: Primary events and the trapping of energy. In: Bioenergetics of Photosynthesis (Ed. Govindjee). Academic Press, New York, San Francisco, London. pp. 115-181 (1975)

26. Simpson, D.J., O. VALLON \& D. von WetTSTEIN: Freeze-fracture studies on barley plastid membranes VIII. In viridis- ${ }^{115}$, a mutant completely lacking photosystem II, OEE 1 and the $\alpha$-subunit of cytochrome $b$-559 accumulate in appressed thylakoids. (In preparation)

27. SiMPSON, D.J. \& D. vON WETTSTEIN: Macromolecu- 
lar physiology of plastids XIV. Viridis mutants in barley: genetic, fluoroscopic and ultrastructural characterisation. Carlsberg Res. Commun. 45, 211-244 (1980)

28. Van Dorssen, R.J., J.J. PliJter, J.P. Dekker, A. DEN OUDEN. J. AMESZ \& H.J. vAN GORKOM: Spectrophotometric properties of chloroplast grana membranes and of the core of Photosystem II. Biochim. Biophys. Acta 890, 134-143 (1987)

Accepted by E. Lund 